\title{
TWISTED CONJUGACY CLASSES IN SYMPLECTIC GROUPS, MAPPING CLASS GROUPS AND BRAID GROUPS (INCLUDING AN APPENDIX WRITTEN WITH FRANCOIS DAHMANI)
}

\author{
ALEXANDER FEL'SHTYN AND DACIBERG L. GONÇALVES \\ ABstract. We prove that the symplectic group $S p(2 n, \mathbb{Z})$ and the map- \\ ping class group $\operatorname{Mod}_{S}$ of a compact surface $S$ satisfy the $R_{\infty}$ property. \\ We also show that $B_{n}(S)$, the full braid group on $n$-strings of a surface \\ $S$, satisfies the $R_{\infty}$ property in the cases where $S$ is either the compact \\ disk $D$, or the sphere $S^{2}$. This means that for any automorphism $\phi$ of $G$, \\ where $G$ is one of the above groups, the number of twisted $\phi$-conjugacy \\ classes is infinite.
}

\section{Contents}

1. Introduction

2. Preliminaries

3. Automorphisms of the symplectic group

4. Automorphisms of the mapping class group

5. Automorphisms of the braid groups of $S^{2}$ and disk $D$

6. Appendix: Geometric group theory and $R_{\infty}$ property for mapping class group

References

Date: February 1, 2008.

2000 Mathematics Subject Classification. 20E45;37C25; 55M20.

Key words and phrases. Reidemeister number, twisted conjugacy classes, Braids group, Mapping class group, Symplectic group.

This work was initiated during our visit to the University of British Columbia, Vancouver in April 2007 and the visit of the first author to the Université Paul Sabatier, Toulouse in May-June 2007 and was completed during conferences in Bendlewo and Warsaw in July-August 2007. 


\section{INTRODUCTION}

Let $\phi: G \rightarrow G$ be an automorphism of a group $G$. A class of equivalence relation defined by $x \sim g x \phi\left(g^{-1}\right)$ is called the Reidemeister class, $\phi$-conjugacy class, or twisted conjugacy class of $\phi$. The number $R(\phi)$ of Reidemeister classes is called the Reidemeister number of $\phi$. The interest in twisted conjugacy relations has its origins, in particular, in the NielsenReidemeister fixed point theory (see, e.g. [43, 35, 12]), in Selberg theory (see, eg. [46, 1]), and in Algebraic Geometry (see, e.g. [30]).

A current significant problem in this area is to obtain a twisted analogue of the Burnside-Frobenius theorem [16, 12, 19, 20, 49, 18, 17, that is, to show the coincidence of the Reidemeister number of $\phi$ and the number of fixed points of the induced homeomorphism of an appropriate dual object. One step in this process is to describe the class of groups $G$ for which $R(\phi)=\infty$ for any automorphism $\phi: G \rightarrow G$.

The work of discovering which groups belong to this class of groups was begun by Fel'shtyn and Hill in [16. It was later shown by various authors that the following groups belong to this class: (1) non-elementary Gromov hyperbolic groups [13, 39]; (2) Baumslag-Solitar groups $B S(m, n)=$ $\left\langle a, b \mid b a^{m} b^{-1}=a^{n}\right\rangle$ except for $B S(1,1)$ [14]; (3) generalized Baumslag-Solitar groups, that is, finitely generated groups which act on a tree with all edge and vertex stabilizers infinite cyclic [38]; (4) lamplighter groups $\mathbb{Z}_{n} \prec \mathbb{Z}$ if and only if $2 \mid n$ or $3 \mid n$ [27]; (5) the solvable generalization $\Gamma$ of $B S(1, n)$ given by the short exact sequence $1 \rightarrow \mathbb{Z}\left[\frac{1}{n}\right] \rightarrow \Gamma \rightarrow \mathbb{Z}^{k} \rightarrow 1$ as well as any group quasi-isometric to $\Gamma$ [47], such groups are quasi-isometric to $B S(1, n)$ [48] ( note however that the class of groups for which $R(\phi)=\infty$ for any automorphism $\phi$ is not closed under quasi-isometry); (6) saturated weakly branch groups, including the Grigorchuk group and the Gupta-Sidki group [21]; (7)The R. Thompson group F [4].

The paper [47] suggests a terminology for this property, which we would like to follow. Namely, a group $G$ has property $R_{\infty}$ if all of its automorphisms $\phi$ satisfy $R(\phi)=\infty$.

For the immediate consequences of the $R_{\infty}$ property in topological fixed point theory see, e.g., [48]. 
In the present paper we prove that the symplectic group $S p(2 n, \mathbb{Z})$ and the mapping class group $\operatorname{Mod}_{S}$ of a compact surface $S$ have the $R_{\infty}$ property. We also show that $B_{n}(S)$, the full braid group on $n$-strings of a compact surface $S$, satisfy the $R_{\infty}$ property in the cases where $S$ is either the compact disk $D$, or the sphere $S^{2}$. The results of the present paper indicate that the further study of Reidemeister theory for these groups should go along the lines similar to those of the infinite case. On the other hand, these results reduces the class of groups for which the twisted Burnside-Frobenius conjecture [16, 19, 20, 49, 18, 17] has yet to be verified.

The paper is organized into 6 sections. In section 2 we describe a very naive procedure to decide whether a group has the $R_{\infty}$ property. Also we recall some known relations between mapping class groups and braid groups which will be used later. In section 3 we show that the symplectic group has the $R_{\infty}$ property. In section 4 we give algebraic proof of the $R_{\infty}$ property for the Mapping class group of the closed surfaces. There we use the short exact sequence

$$
1 \rightarrow I_{n} \rightarrow \operatorname{Mod}_{S} \rightarrow S p(2 n, \mathbb{Z}) \rightarrow 1
$$

where $I_{n}$ is the Torelli group. In section 5 we show that, with a few exceptions, the braids groups of the disk $D$ and the sphere $S^{2}$ have the $R_{\infty}$ property.

In Appendix, written with Francois Dahmani, we use geometric methods to show that the mapping class group of the compact surfaces has the $R_{\infty}$ property with few obvious exceptions.

Acknowledgments The first author would like to thank V. Guirardel, J. Guaschi, M. Kapovich and J.D. McCarthy for stimulating discussions and comments. He also thanks the Université Paul Sabatier, Toulouse for its kind hospitality and support while the part of this work has been completed.

\section{Preliminaries}

Consider a group extension respecting the homomorphism $\phi$ : 


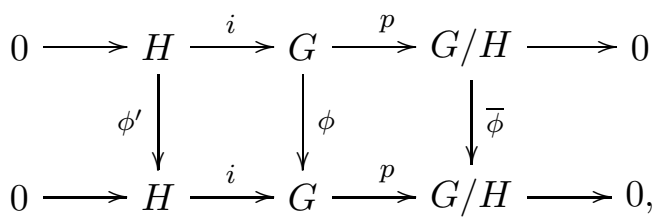

where $H$ is a normal subgroup of $G$. First, let us remark that the Reidemeister classes of $\phi$ in $G$ are mapped epimorphically on classes of $\bar{\phi}$ in $G / H$. Indeed,

$$
p(\widetilde{g}) p(g) \bar{\phi}\left(p\left(\widetilde{g}^{-1}\right)\right)=p\left(\widetilde{g} g \phi\left(\widetilde{g}^{-1}\right)\right.
$$

Moreover, if $R(\phi)<\infty$, then the previous remark implies $R(\bar{\phi})<\infty$.

We next give a criterion for a group to have the $R_{\infty}$ property.

Lemma 2.1 Suppose that a group $G$ has an infinite number of conjugacy classes. Then any inner automorphism also has infinite Reidemeister number. Moreover, if $\left\{\phi_{j}\right\}_{j \in O u t(G)}$ is a subset of $\operatorname{Aut}(G)$ which contains single representatives for each coset $\operatorname{Aut}(G) / \operatorname{Inn}(G)$ and $R\left(\phi_{j}\right)=\infty$ for all $j$, then $G$ has the $R_{\infty}$ property.

Proof. The first part of the statement was proved in [4]. The second part follows easily using a similar argument which we now recall. Let $\phi$ be an automorphism and $\theta$ an element of the group. Then we have two automorphisms, namely $\phi$ and the composite of $\phi$ with the inner automorphism which is conjugation by $\theta$, which we denote by $\theta \circ \phi$. We claim that multiplication by $\theta^{-1}$ on the right provides a bijection between the set of Reidemeister classes of $\phi$ and those of $\theta \circ \phi$. Consider two elements where the first is denoted by $\alpha$ and the second is of the form $\beta \alpha \phi(\beta)^{-1}$ for some $\beta \in G$. We claim that the two elements $\alpha \theta^{-1}$ and the $\beta \alpha \phi(\beta)^{-1} \theta^{-1}$ are in the same $\theta \circ \phi$ Reidemeister classes. To show this, we write class of $\alpha \theta^{-1}$ as $\beta \alpha \theta^{-1}\left((\theta \circ \phi(\beta))^{-1}\right)=\beta \alpha \theta^{-1} \theta \phi(\beta)^{-1} \theta^{-1}=\beta \alpha \phi(\beta)^{-1} \theta^{-1}$. Thus $\alpha \theta^{-1}$ and $\beta \alpha \phi(\beta)^{-1} \theta^{-1}$ are in the same Reidemeister class of $\theta \circ \phi$. Similarly, multiplication by $\theta$ on the right provides a bijection between the set of Reidemeister classes of $\theta \circ \phi$ and those of $\phi$. One correspondence is the inverse of the other and the result follows. 
Lemma 2.2 Let $\phi \in \operatorname{Aut}(G)$ and $Q_{i}$ be an infinite family of quotients of $G$,

$$
1 \rightarrow K_{i} \rightarrow G \rightarrow Q_{i} \rightarrow 1
$$

such that $\phi\left(K_{i}\right) \subset K_{i}$. If the sequence of numbers $R\left(\phi_{i}\right)$ is unbounded, where $\phi_{i}$ is the induced homomorphism on the quotient $Q_{i}$, then $R(\phi)=\infty$.

Proof. Since $p: G \rightarrow Q_{i}$ is surjective, we have $R(\phi) \geq R\left(\phi_{i}\right)$. If $R(\phi)$ is finite then the sequence $R\left(\phi_{i}\right)$ is bounded. Hence the result follows.

Despite the fact that the Lemma 2.2 is quite obvious, it is usefull for its ability to find such quotients and to estimate Reidemeister number on the quotient. We will apply Lemma 2.2 in a situation where the quotients are finite groups.

We will now state a result which relates braid groups and mapping class groups. We will use this relation to study the braid group of the sphere. Following section 2 of [45], let $S$ be a 2-manifold with $n$ distinguished points in its interior. Let $H(S, n)$ denotes the space of homeomorphisms of $S$, which fix pointwise the $n$ distinguished points. If $n=0$, we set $H(S)=H(S, 0)$. We define $G(S, n)=\pi_{0}(H(S, n))$ and, if $n=0$, we set $G(S)=G(S, 0)$.

If $S$ is the sphere $S^{2}$ we have (see the bottom of the page 615 in [45]):

Theorem 2.3 If $S$ is $S^{2}$ then we have the short exact sequence

$$
1 \rightarrow \mathbb{Z}_{2} \rightarrow B_{r}\left(S^{2}\right) \rightarrow G\left(S_{r}^{2}\right) \rightarrow G\left(S^{2}\right) \rightarrow 1
$$

where $S_{r}^{2}=S^{2}-r$ open disks.

It is well known that $G\left(S^{2}\right)=\mathbb{Z}_{2}$, where the nontrivial element is represented by the isotopy class of an orientation reversing homeomorphism of the sphere. So the preimage of the element $i d \in G\left(S^{2}\right)$ with respect to the projection $G\left(S_{r}^{2}\right) \rightarrow G\left(S^{2}\right)$ gives the mappping class group $\operatorname{Mod}_{S_{r}^{2}}$ (see section 4 for the definition of $\operatorname{Mod}_{S}$ ). The above sequence implies immediately the following short exact sequence:

$$
1 \rightarrow \mathbb{Z}_{2} \rightarrow B_{r}\left(S^{2}\right) \rightarrow \operatorname{Mod}_{S_{r}^{2}} \rightarrow 1
$$

This sequence will used in section 5 . 


\section{Automorphisms of THE SYMPLECTIC GROUP}

Let $S p(2 n, \mathbb{Z})$ denote the symplectic group over the integers. This group is sometimes called proper symplectic group as in [40]. For some choice of a basis, the group $S p(2 n, \mathbb{Z})$ may be defined as the set of matrices $2 n \times 2 n$ over $\mathbb{Z}$ which satisfy the following conditions: for all $1 \leq l<j \leq 2 n$, $\sum_{i=1}^{i=n}\left(a_{2 i-1, l} a_{2 i, j}-a_{2 i-1, j} a_{2 i, l}\right)$ is equal 1 if $(l, j)$ is of the form $(2 k-1,2 k)$ and is equal to 0 otherwise. In [40] and [42] the description of the symplectic group is somewhat different to the one given above. One definition is given in terms of a basis and the other is given in terms of a basis which corresponds to a permutation of the first base. Recall that the elements of the the group $S p(2 n, \mathbb{Z})$ are automorphisms which are obtained as the induced homomorphisms in $H^{1}(S, \mathbb{Z})$ by an orientation preserving homeomorphisms of the orientable closed surface $S$ of genus $n$.

We refer to [42] and [44] for most of the properties of the group of symplectic matrices. The study of the Reidemeiser number of automorphism of the unimodular groups is in progress, while the corresponding basic material may be found in 32 ] and 42 .

Following [44] let us call a homomorphism of $S p(2 n, \mathbb{Z})$ into $\{ \pm 1\}$ a character. It is known that every automorphism $\phi$ of $S p(2 n, \mathbb{Z})$ is given by $\phi(M)=\psi(M) U M U^{-1}$ for all $M \in S p(2 n, \mathbb{Z})$, where $\psi$ is a character and $U$ is a matrix in the full symplectic group. We firstly show that, if we change an automorphism by a character, then we do not change the finiteness of the Reidemeister number of the resulting automorphism. Thus we reduce the problem to the same problem for the automorphisms given by conjugation by the elements of the full symplectic group. Since the proper sympletic group has index 2 in the full symplectic group, then, by the Lemma 2.1, we reduce our problem to the study of the finiteness of Reidemeister number of ONE automorphism given as conjugation by one element of the full symplectic group, which is not in the proper symplectic group. We also need to know the number of usual conjugacy classes in the group.

Lemma 3.1 Let $\phi: S p(2 n, \mathbb{Z}) \rightarrow S p(2 n, \mathbb{Z})$ be an automorphism and $\psi: S p(2 n, \mathbb{Z}) \rightarrow \mathbb{Z}_{2}$ be a character. $R(\phi)$ is finite if and only if $R(\psi \cdot \phi)$ is finite, where homomorphism $\psi \cdot \phi$ is given by $(\psi \cdot \phi)(g)=\psi(g) \cdot \phi(g)$ for all $g \in S p(2 n, \mathbb{Z})$. 
Proof. If $\psi$ is the trivial homomorphism then the result is clear since the two homomorphisms are the same. Otherwise, let $H$ be the kernel of $\psi$, which is a subgroup of index 2 in $S p(2 n, \mathbb{Z})$. We split each Reidemeister class of $\phi$ into two disjoint subsets, namely two elements $y_{1}, y_{2}$ of a Reidemeister class are in the same subset if and only if $y_{2}=a y_{1} \phi(a)^{-1}$ for some $a \in H$. It is straightforward to see that this relation gives a partition of a Reidemeister class into two nonempty subsets. The set all these subsets as we run over the set of all Reidemeister classes of $\phi$ gives a partition of the group. Now a Reidemeister class of the homomorphism $\psi \cdot \phi$ which contains an element $y$ is the union of the subset containing $y$ with the subset containing the element $a y \phi(a)^{-1}$ for some element $a$ which does not belong to $H$. This implies the result.

Let $\phi$ be the automorphism which is the conjugation by the diagonal matrix of order $2 n$, where the elements of the diagonal are $a_{i, i}=(-1)^{i+1}$. It is easy to verify that if $M=\left(m_{i, j}\right)$ then $\phi(M)_{i, j}=(-1)^{i+j} m_{i, j}$.

Now we prove the result for the case $n=1$. The proof of the general case will follow the same strategy as in this case and will eventually reduce to the case $n=1$.

Proposition 3.2 If $\phi: S p(2, \mathbb{Z}) \rightarrow S p(2, \mathbb{Z})$ is an automorphism, then $R(\phi)=\infty$.

Proof. Let $p$ be a prime. The set of invertible elements in the cyclic group $\mathbb{Z}_{p}$ is a multiplicative group of order $p-1$ denoted by $\mathbb{Z}_{p}^{*}$. The number of elements of the set $V_{1}=\left\{w \in \mathbb{Z}_{p}^{*} \mid w^{2}=-1\right\}$ is at most 2. If $p-1$ is divisible by 4 then this set has 2 elements, otherwise it is empty. Consider the groups $S p\left(2, \mathbb{Z}_{p}\right)$, the Symplectic group module the prime $p$, when $p$ runs over the set of all primes. The groups $S p\left(2, \mathbb{Z}_{p}\right)$ are finite. We will use Lemma 2.2 to obtain information about the automorphism $\phi$. The automorphism $\phi$ sends the matrix

$$
\left(\begin{array}{ll}
a & b \\
c & d
\end{array}\right)
$$

to

$$
\left(\begin{array}{cc}
a & -b \\
-c & d
\end{array}\right) .
$$


The order of the group $S p\left(2, \mathbb{Z}_{p}\right)$ is $p\left(p^{2}-1\right)$. This group contains a subgroup isomorphic to $Z_{p-1}$. Consider the subgroup generated by the diagonal matrices of the form

$$
\left(\begin{array}{cc}
w & 0 \\
0 & w^{-1}
\end{array}\right)
$$

where $0 \neq w \in \mathbb{Z}_{p}$. Denote by $\bar{w}$ the above matrix determined by the element $w$. We now compute the elements of this subgroup which are in the same Reidemeister class of $\bar{w}$ for $w$ not in $V_{1}$. We claim that $\bar{w}$ and $-\bar{w}^{-1}$ are the only elements in the Reidemeister class of $\bar{w}$. This will imply that the number of Reidemeister classes of $\phi$ is at least $(p-3) / 2$. For the calculation of the elements of the subgroup which are in the Reidemeister class of $\bar{w}$ let us take an arbitrary matrix $M$ in $S p\left(2, \mathbb{Z}_{p}\right)$ and let us require that $M \bar{w} \phi\left(M^{-1}\right)$ is diagonal. If the Symplectic matrix $M$ is given by

$$
\left(\begin{array}{ll}
a & b \\
c & d
\end{array}\right)
$$

then $\phi(M)$ is given by

$$
\left(\begin{array}{cc}
a & -b \\
-c & d
\end{array}\right)
$$

and $\phi\left(M^{-1}\right)$ is equal to

$$
\left(\begin{array}{ll}
d & b \\
c & a
\end{array}\right)
$$

and the product $M \bar{w} \phi\left(M^{-1}\right)$ is equal to

$$
\left(\begin{array}{cc}
w a d+w^{-1} b c & w a b+w^{-1} a b \\
w c d+w^{-1} c d & w b c+w^{-1} a d
\end{array}\right) .
$$

The above matrix is diagonal if and only if $\left(w+w^{-1}\right) a b=0$ and $(w+$ $\left.w^{-1}\right) c d=0$. Since $w+w^{-1} \neq 0$ because $w$ does not belong to $V_{1}$, we must have $a b=0$ and $c d=0$. Since $M$ has determinant 1 , the possible solutions are: 1) $a=0$ and $d=0$ or 2) $b=0$ and $c=0$. In the first case we have $b c=-1$ and the diagonal elements are $-w^{-1}$ and $-w$. In the second case we have $a b=1$ and the diagonal elements are $w$ and $w^{-1}$. So each Reidemeister 
class contains 2 elements of this form. So the number of Reidemeister classes is at most $(p-3) / 2$ and the result follows.

Now we prove the main result of this section.

Theorem 3.3 If $\phi: S p(2 n, \mathbb{Z}) \rightarrow S p(2 n, \mathbb{Z})$ is an automorphism, then $R(\phi)=\infty$.

Proof. We will reduce the problem to the case of $\operatorname{Sp}(2, \mathbb{Z})$ at the very end. Let $M \in S p(2 n, \mathbb{Z})$ be given by

$$
\left(\begin{array}{ccccc}
a_{1,1} & a_{1,2} & \ldots . & a_{1,2 n-1} & a_{1,2 n} \\
b_{1,1} & b_{1,2} & \ldots & b_{1,2 n-1} & b_{1,2 n} \\
\cdot & \cdot & \cdot & \cdot & \\
\cdot & \cdot & \cdot & \cdot & \\
a_{n, 1} & a_{n, 2} & \ldots . & a_{n, 2 n-1} & a_{n, 2 n} \\
b_{n, 1} & b_{n, 2} & \ldots . & b_{n, 2 n-1} & b_{n, 2 n}
\end{array}\right) .
$$

Then $\phi(M)^{-1}$ is given by

$$
\left(\begin{array}{ccccc}
b_{1,2} & a_{1,2} & \ldots . & b_{n, 2} & a_{n, 2} \\
b_{1,1} & a_{1,1} & \ldots . & b_{n, 1} & a_{n, 1} \\
\cdot & \cdot & \cdot & \cdot & \\
\cdot & \cdot & \cdot & \cdot & \\
b_{1,2 n} & a_{1,2 n} & \ldots . & b_{n, 2 n} & a_{n, 2 n} \\
b_{1,2 n-1} & a_{1,2 n-1} & \ldots . & b_{n, 2 n-1} & a_{n, 2 n-1}
\end{array}\right) .
$$

Let $\bar{w}$ be the matrix

$$
\left(\begin{array}{ccccc}
w & 0 & \ldots . & 0 & 0 \\
0 & w^{-1} & \ldots & 0 & 0 \\
\cdot & \cdot & \cdot & \cdot & \\
\cdot & \cdot & \cdot & & \\
0 & 0 & \ldots . & 1 & 0 \\
0 & 0 & \ldots \ldots & 0 & 1
\end{array}\right) .
$$

In order to describe $M \bar{w} \phi\left(M^{-1}\right)$, let us introduce the following notation. Let

$$
A_{2 i-1,2 j-1}=w^{\epsilon} a_{i, 1} b_{j, 2}+w^{-\epsilon} b_{j, 1} a_{i, 2} . .+. . a_{i, 2 n-1} b_{j, 2 n}+a_{i, 2 n} b_{j, 2 n-1},
$$




$$
\begin{gathered}
A_{2 i-1,2 j}=w^{\epsilon} a_{i, 2} a_{i, 1}+w^{-\epsilon} a_{i, 2} a_{i, 1} . .+. . a_{i, 2 n-1} a_{i, 2 n}+a_{i, 2 n} a_{i, 2 n-1}, \\
A_{2 i, 2 j-1}=w^{\epsilon} b_{j, 1} b_{j, 2}+w^{-\epsilon} b_{j, 1} b_{j, 2} .+. . b_{j, 2 n-1} b_{j, 2 n}+b_{j, 2 n} b_{j, 2 n-1} \\
A_{2 i, 2 j}=w b_{j, 1} a_{i, 2}+w^{-1} b_{j, 2} a_{i, 1} . .+. . b_{j, 2 n-1} a_{i, 2 n}+b_{j, 2 n} a_{i, 2 n-1} .
\end{gathered}
$$

where $\epsilon=1$ for $j=1,2$ and zero otherwise.

The product $M \bar{w} \phi\left(M^{-1}\right)$ is given by

$$
\left(\begin{array}{ccccc}
A_{1,1} & A_{1,2} & \ldots . & A_{1,2 n-1} & A_{1,2 n} \\
A_{2,1} & A_{2,2} & \ldots \ldots & A_{2,2 n-1} & A_{2,2 n} \\
\ldots & \ldots & \ldots & \ldots & \\
\ldots & \ldots & \ldots & \ldots & \\
A_{2 n-1,1} & A_{2 n-1,2} & \ldots \ldots & A_{2 n-1,2 n-1} & A_{2 n-1,2 n} \\
A_{2 n, 1} & A_{2 n, 2} & \ldots \ldots & A_{2 n, 2 n-1} & A_{2 n, 2 n}
\end{array}\right) .
$$

It follows that $w a_{i, 1} b_{j, 2}+w^{-1} b_{j, 1} a_{i, 2} . .+. . a_{i, 2 l-1} b_{j, 2 l}+a_{i, 2 l} b_{j, 2 l-1} . .+. . a_{i, 2 n-1} b_{j, 2 n}+$ $a_{i, 2 n} b_{j, 2 n-1}=w a_{i, 2} b_{j, 1}+w^{-1} b_{j, 2} a_{i, 1} . .+. . a_{i, 2 l} b_{j, 2 l-1}+a_{i, 2 l-1} b_{1,2 l} . .+. . a_{i, 2 n} b_{j, 2 n-1}+$ $a_{i, 2 n-1} b_{j, 2 n}=0$ for all $(i, j) \neq(1.1), 1 \leq i, j \leq n$. This implies the system of equations: $\left(w-w^{-1}\right) b_{j, 2} a_{i, 1}=\left(w-w^{-1}\right) b_{j, 1} a_{i, 2}$ for all $(i, j) \neq(1,1)$, $1 \leq i, j \leq n$. So all $2 \times 2$ submatrices of the matrix whose columns are $a_{1,1}, b_{1,1}, \ldots a_{n, 1}, b_{n, 1}$ for $1 \leq i \leq n$ and $a_{1,2}, b_{1,2}, \ldots . a_{n, 2}, b_{n, 2}$ for $1 \leq i \leq n$ $b_{i, 1}$ for $1 \leq i \leq n$ different from

$$
\left(\begin{array}{ll}
a_{1,1} & b_{1,1} \\
a_{2,1} & b_{2,1}
\end{array}\right)
$$

have determinant zero. This implies that the above $2 \times 2$ matrix has determinant 1 ( so is not zero) and $a_{i, 1}=a_{i, 2}=b_{i, 1}=b_{i, 2}=0$ for $i>1$.

So the matrices $M$ and $\bar{w} \phi(M)^{-1}$ are respectively of the form

$$
\left(\begin{array}{ccccccc}
a_{1,1} & a_{1,2} & a_{1,3} & a_{1,4} & \ldots . . & a_{1,2 n-1} & a_{1,2 n} \\
b_{1,1} & b_{1,2} & b_{1,3} & b_{1,4} & \ldots . & b_{1,2 n-1} & b_{1,2 n} \\
0 & 0 & a_{2,3} & a_{2,4} & \ldots . . & a_{2,2 n-1} & a_{2,2 n} \\
0 & 0 & b_{2,3} & b_{2,4} & \ldots . . & b_{2,2 n-1} & b_{2,2 n} \\
. & . & . & . & \ldots . & . & . \\
0 & 0 & a_{n, 3} & a_{n, 4} & \ldots . . & a_{n, 2 n-1} & a_{n, 2 n} \\
0 & 0 & b_{n, 3} & b_{n, 4} & \ldots . . & b_{n, 2 n-1} & b_{n, 2 n}
\end{array}\right) .
$$

and 


$$
\left(\begin{array}{ccccccc}
w b_{1,2} & w^{-1} a_{1,2} & 0 & 0 & \ldots . & 0 & 0 \\
w b_{1,1} & w^{-1} a_{1,1} & 0 & 0 & \ldots . . & 0 & 0 \\
b_{1,4} & a_{1,4} & b_{2,4} & a_{2,4} & \ldots . & b_{n, 4} & a_{n, 4} \\
b_{1,3} & a_{1,3} & b_{2,3} & a_{23} & \ldots \ldots & b_{n, 3} & a_{n, 3} \\
\cdot & \cdot & \cdot & \cdot & \ldots \ldots & \cdot & \cdot \\
\cdot & \cdot & \cdot & \cdot & \ldots \ldots & \cdot & \cdot \\
b_{1,2 n} & a_{1,2 n} & b_{2,2 n} & a_{2,2 n} & \ldots \ldots & b_{n, 2 n} & a_{n, 2 n} \\
b_{1,2 n-1} & a_{1,2 n-1} & b_{2,2 n-1} & a_{2,2 n-1} & \ldots \ldots & b_{n, 2 n-1} & a_{n, 2 n-1}
\end{array}\right) .
$$

The product $M \bar{w}\left(\phi(M)^{-1}\right.$ is of the form

$$
\left(\begin{array}{cc}
A & 0 \\
0 & I_{2 n-2}
\end{array}\right)
$$

where the $\mathrm{A}$ is of order $2 \times 2, I_{2 n-2}$ is the identity matrix of order $2 n-2$, and $0^{\prime} s$ are the trivial matrices of orders $2 \times 2 n-2,2 n-2 \times 2$, respectively. Hence the submatrix of order $2 n-2 \times 2 n-2$

$$
\left(\begin{array}{ccccc}
b_{2,4} & a_{2,4} & \ldots \ldots & b_{n, 4} & a_{n, 4} \\
b_{2,3} & a_{4,3} & \ldots \ldots & b_{n, 3} & a_{n, 3} \\
\cdot & \cdot & \ldots \ldots & \cdot & \cdot \\
\cdot & \cdot & \ldots \ldots & \cdot & \cdot \\
b_{2,2 n} & a_{2,2 n} & \ldots \ldots & b_{n, 2 n} & a_{n, 2 n} \\
b_{2,2 n-1} & a_{2,2 n-1} & \ldots \ldots & b_{n, 2 n-1} & a_{n, 2 n-1}
\end{array}\right)
$$

is invertible. Regarding the columns as a vector in the $2 \mathrm{n}-2$ dimensional vector space over the rationals, these $2 \mathrm{n}-2$ vectors form a basis. If $v$ is any of the above column, then the inner product of $\left(a_{1,1}, a_{1,2}, a_{1,3}, a_{1,4}, \ldots . ., a_{1,2 n-1}, a_{1,2 n}\right)$ with the column vector $(0,0, v)$ is zero. Therefore the inner product of $\left(a_{1,3}, a_{1,4}, \ldots . ., a_{1,2 n-1}, a_{1,2 n}\right)$ and $v$ is also zero for all $v$. Since the set of all $v^{\prime} s$ forms a basis, this implies that $\left(a_{1,3}, a_{1,4}, \ldots . ., a_{1,2 n-1}, a_{1,2 n}\right)$ is trivial and 
the matrices $M$ and $\bar{\phi}(M)^{-1}$ are of the form

$$
\left(\begin{array}{ccccccc}
a_{1,1} & a_{1,2} & 0 & 0 & \ldots \ldots & 0 & 0 \\
b_{1,1} & b_{1,2} & 0 & 0 & \ldots \ldots & 0 & 0 \\
0 & 0 & a_{2,3} & a_{2,4} & \ldots \ldots & a_{2,2 n-1} & a_{2,2 n} \\
0 & 0 & b_{2,3} & b_{2,4} & \ldots . . & b_{2,2 n-1} & b_{2,2 n} \\
. & . & . & . & \ldots \ldots & . & . \\
0 & 0 & a_{n, 3} & a_{n, 4} & \ldots \ldots & a_{n, 2 n-1} & a_{n, 2 n} \\
0 & 0 & b_{n, 3} & b_{n, 4} & \ldots . . & b_{n, 2 n-1} & b_{n, 2 n}
\end{array}\right)
$$

and

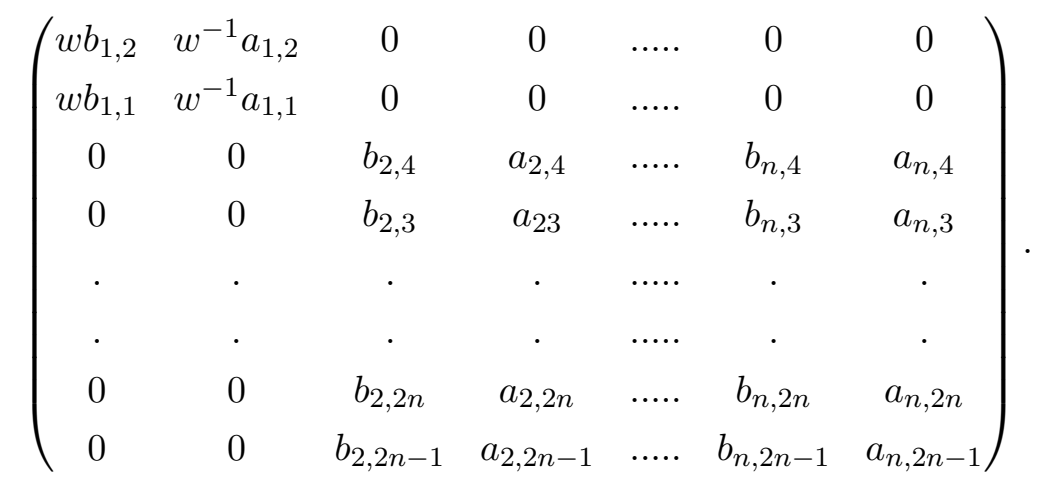

The $2 \times 2$ submatrix $A$ of the product $M \bar{w}\left(\phi(M)^{-1}\right.$ is given as in the case when $n=1$. Thus we will have as solution for $a_{1,1}, a_{1,2}, b_{1,1}, b_{1,2}$ the same solutions as in the case $n=1$ and the result follows.

\section{Automorphisms of the mapping Class group}

In this section we study the $R_{\infty}$ property for the Mapping class group of a closed surface. As an application of the results of the previous section, we show that mapping class group of a closed orientable surface of genus $g>0$ has the $R_{\infty}$ property. We start by quoting some results about 
Outer automorphism group of the mapping class group. Let $S$ be a compact orientable surface. Recall that $\operatorname{Mod}_{S}$ denotes the group of orientationpreserving homeomorphisms of $S$ modulo isotopy, and $\operatorname{Mod}_{S}^{*}$ denotes the group of the homeomorphisms of $S$ modulo isotopies. From [34] we have

Theorem 4.1 If $S$ is neither a sphere with $\leq 4$ holes, nor a torus with $\leq 2$ holes, nor a closed surface of genus 2, then every automorphism of $\operatorname{Mod}_{S}$ is given by the restriction of an inner automorphism of $\operatorname{Mod}_{S}^{*}$. In particular $\operatorname{Out}\left(M_{S}\right)$ is a finite group and moreover,

$$
\operatorname{Out}\left(\operatorname{Mod}_{S}\right)=Z_{2}, \operatorname{Out}\left(\operatorname{Mod}_{S}^{*}\right)=1 .
$$

Theorem 4.2 If $S$ is a closed surface of genus 2 then $\operatorname{Out}\left(M_{S}\right)$ is canonically isomorphic to $H^{1}\left(\operatorname{Mod}_{S} ; Z_{2}\right) \oplus \operatorname{Mod}_{S}^{*} / \operatorname{Mod}_{S}=Z_{2} \oplus Z_{2}$ and $\operatorname{Out}\left(M_{S}^{*}\right)$ is canonically isomorphic to $H^{1}\left(\operatorname{Mod}_{S}^{*} ; Z_{2}\right)=Z_{2} \oplus Z_{2}$.

In 34 the remaining cases also are given. In the cases where $S$ is either the torus $T$ or the torus with one hole we have $\operatorname{Mod}_{S}=S l_{2}(\mathbb{Z})$ and $\operatorname{Mod}_{S}^{*}=$ $G l_{2}(\mathbb{Z})$, so $\operatorname{Out}\left(\operatorname{Mod}_{S}^{*}\right)=1$ and $\operatorname{Out}\left(\operatorname{Mod}_{S}\right)=Z_{2}$. For $S=S^{2}$ we have $\operatorname{Mod}_{S}=\{1\}$, the trivial group, and $\operatorname{Mod}_{S}^{*}=\mathbb{Z}_{2}$, therefore $\operatorname{Out}\left(\operatorname{Mod}_{S}^{*}\right)=$ $\operatorname{Out}\left(\operatorname{Mod}_{S}\right)=1$.

We will show that the mapping class group of closed surface has the $R_{\infty}$ property.

Theorem 4.3 Let $S$ be an orientable closed surface. The mapping class group $\operatorname{Mod}_{S}$ has the $R_{\infty}$ property if and only if $S$ is not $S^{2}$.

Proof. If $S$ is $S^{2}$ we know that the mapping class group is finite. If $S=T$ then $\operatorname{Mod}_{S}=S l_{2}(\mathbb{Z})$ and this group has the $R_{\infty}$ property. In the remaining cases, we consider the short exact sequence

$$
1 \rightarrow I_{n} \rightarrow \operatorname{Mod}_{S} \rightarrow S p(2 n, \mathbb{Z}) \rightarrow 1,
$$

where $I_{n}$ is the kernel of the well- known homomorphism $p: \operatorname{Mod}_{S} \rightarrow$ $S p(2 n, \mathbb{Z})$, also known as the Torelli group.

If the genus $g>2$, then we consider an automorphism $\phi$ which represents the nontrivial element of $\operatorname{Out}\left(\operatorname{Mod}_{S}\right)=Z_{2}$. Pick an automorphism $\phi$ which is conjugation by an element of the group $\operatorname{Mod}_{S}^{*}$ represented by a orientation reversing homeomorphism of surface $S$. It preserves the Torelli group, and so we obtain a homomorphism of the short exact sequence. Thus we obtain an induced automorphism $\bar{\phi}$ in the quotient group $S p(2 n, \mathbb{Z})$. 
The Reidemeister number $R(\bar{\phi})$ is infinite by Theorem 3.3. Then remark about extensions (2.1) implies that the Reidemeister number $R(\phi)$ is also infinite. Then the $R_{\infty}$ property follows from Lemma 2.1. For genus $g=2$, we have to show that 3 automorphisms of $\operatorname{Mod}_{S}$ have infinite numbers of twisted conjugacy classes, since $\operatorname{Out}(\operatorname{Mod} S)=\mathbb{Z}_{2}+\mathbb{Z}_{2}$ in this case. These three automorphisms are all conjugation, so they preserve the Torelli group and the result follows as above.

\section{Automorphisms of the BRaid groups of $S^{2}$ AND Disk $D$}

We prove in this section the $R_{\infty}$ property for $B_{n}\left(S^{2}\right)$. This case will be investigated using the results of the previous section and the relation between mapping class groups and braids groups given in section 2 . Then we will consider the case when $S$ is a disk $D$, namely the group $B_{n}(D)$, also called the Artin braid group on $n$-strings.

Theorem 5.1 The group $B_{n}\left(S^{2}\right)$ has the $R_{\infty}$ property if and only if $n>3$.

Proof. Let $\phi: B_{n}\left(S^{2}\right) \rightarrow B_{n}\left(S^{2}\right)$ be an automorphism. Since the center of $B_{n}\left(S^{2}\right)$ is a characteristic subgroup, $\phi$ induces a homomorphism of the short exact sequence

$$
1 \rightarrow \mathbb{Z}_{2} \rightarrow B_{n}\left(S^{2}\right) \rightarrow \operatorname{Mod}_{S_{n}^{2}} \rightarrow 1
$$

where the short exact sequence was obtained from the sequence in Theorem 2.3. The results of Appendix imply that the group $\operatorname{Mod}_{S_{n}^{2}}$, for $n>3$, has the $R_{\infty}$ property. Then the remark about extensions (2.1) implies that the group $B_{n}\left(S^{2}\right)$ also has this property. For $n \leq 3$ the groups $B_{n}\left(S^{2}\right)$ are finite so they do not have the $R_{\infty}$ property.

Remark For $n=4$ the lower central series of $B_{4}\left(S^{2}\right)$ has been studied. The commutator subgroup $\left[B_{4}\left(S^{2}\right), B_{4}\left(S^{2}\right)\right]$ is isomorphic to $Q_{8} \rtimes F_{2}(x, y)$, the semi-direct product of the quatenionic group with the free group on two generators [24], whose Abelinization is $\mathbb{Z}_{6}$. Since the $\left[B_{4}\left(S^{2}\right), B_{4}\left(S^{2}\right)\right]$ is characteristic, to show that $B_{4}\left(S^{2}\right)$ has the $R_{\infty}$ property, it is sufficient to show that $\left[B_{4}\left(S^{2}\right), B_{4}\left(S^{2}\right)\right] \simeq Q_{8} \rtimes F_{2}(x, y)$ has $R_{\infty}$ property. But, using 
the remark about extensions (2.1), this follows from the fact that $Q_{8} \subset$ $Q_{8} \rtimes F_{2}(x, y)$ is characteristic and that group $F_{2}(x, y)$ has the $R_{\infty}$ property.

Now let us consider the Artin braid group $B_{n}(D)$. We will show that $B_{n}(D)$ has the $R_{\infty}$ property, except a few cases. It is known from [9] that $\operatorname{Out}\left(B_{n}(D)\right)=\mathbb{Z}_{2}$, but we do not use this fact.

Denote by $\mathbb{B}_{n}(D)$ the quotient of $B_{n}(D)$ by the center.

Proposition 5.2 The group $\mathbb{B}_{n}(D)$ has the $R_{\infty}$ property if and only if $n>2$.

Proof. The "if part" follows from the fact that $B_{1}(D)$ is trivial and $B_{2}(D)$ is isomorphic to $\mathbb{Z}$. So let $n>2$. From Theorem 15 in [9] we know that the free group $F_{n-1}$ of rank $n-1$ is characteristic in $\mathbb{B}_{n}(D)$. From the formulas which relates the Reidemeister classes of the terms of a short exact sequence(see [23]) we conclude that $\mathbb{B}_{n}(D)$ has the $R_{\infty}$ property.

Theorem 5.3 The group $B_{n}(D)$ has the $R_{\infty}$ property if and only if $n>2$.

Proof. The "if part" follows from the fact that $B_{1}(D)$ is trivial and $B_{2}(D)$ is isomorphic to $\mathbb{Z}$. So let $n>2$. Consider the short exact sequence

$$
1 \rightarrow \mathbb{Z} \rightarrow B_{n}(D) \rightarrow \mathbb{B}_{n} \rightarrow 1
$$

Because $\mathbb{Z}$ is the center of $B_{n}(D)$ and the center is characteristic, any automorphism of $B_{n}(D)$ is an automorphism of short exact sequence. Since $\mathbb{B}_{n}$ has the $R_{\infty}$ property the result follows from remark about extensions (2.1).

\section{Appendix: Geometric group theory And $R_{\infty}$ Property For}

\section{MAPPING CLASS GROUP}

The object of this Appendix is to use geometric methods to prove more stronger result then in section 4 . Namely, we show that the known geometric methods apply to prove that for all compact orientable surfaces with genus $g$ and $p$ boundary components, and $3 g+p-4>0$ the mapping class group has the $R_{\infty}$ property with a few exceptions.

We need to use the non-elementary result of Masur and Minsky [41] (see also Bowditch [5]) that the complex of curves of an oriented surface, with 
genus $g$ and $p$ boundary components, and $3 g+p-4>0$, is Gromovhyperbolic space.

Lemma 6.1 If $G$ is a group and $\varphi$ is an endomorphism of $G$, then an element $x \in G$ is always $\varphi$-conjugate to its image $\varphi(x)$.

Proof. Put $\gamma=x^{-1}$. Now $x$ is $\varphi$-conjugate to $x^{-1} x \varphi(x)=\varphi(x)$.

Let $G$ be a group, and $\varphi$ an automorphism of $G$ of order $m$. Construct the group $G_{\varphi}=G \rtimes_{\varphi} \mathbb{Z}_{m}=\left\langle G, t \mid \forall g \in G, \operatorname{tg}^{-1}=\varphi(g), t^{m}=1\right\rangle$.

Lemma 6.2 Two elements $x, y$ of $G$ are $\varphi$-conjugate if and only if $x t$ and $y t$ are conjugate in the usual sense in $G_{\varphi}$. Therefore $R(\varphi)$ is the number of usual conjugacy classes in the $\operatorname{coset} G \cdot t$ of $G$ in $G_{\varphi}$.

Proof. If $x$ and $y$ are $\phi$-conjugate, then there is a $\gamma \in G$ such that $\gamma x=$ $y \varphi(\gamma)$. This implies $\gamma x=y t \gamma t^{-1}$ and therefore $\gamma(x t)=(y t) \gamma$. So $x t$ and $y t$ are conjugate in the usual sense in $G_{\varphi}$. Conversely suppose $x t$ and $y t$ are conjugate in $G_{\varphi}$. Then there is a $\gamma t^{n} \in G_{\varphi}$ with $\gamma t^{n} x t=y t \gamma t^{n}$. From the relation $t x t^{-1}=\varphi(x)$, we obtain $\gamma \varphi^{n}(x) t^{n+1}=y \varphi(\gamma) t^{n+1}$ and therefore $\gamma \varphi^{n}(x)=y \varphi(\gamma)$. This shows that $\varphi^{n}(x)$ and $y$ are $\varphi$-conjugate. However, by lemma $6.1, x$ and $\varphi^{n}(x)$ are $\varphi$-conjugate, so $x$ and $y$ must be $\varphi$-conjugate.

The following lemma was proven by Delzant

Lemma 6.3 [39, Lemma 3.4] If $K$ is a normal subgroup of a group $\Gamma$ acting non-elementary on a hyperbolic space, and if $\Gamma / K$ is abelian, then any coset of $K$ contains infinitely many conjugacy classes.

Proof. Fix $u$ in the coset $C$ under consideration. Suppose for a moment that we can find $c, d \in K$, generating a free group of rank 2, such that $u c^{\infty} \neq c^{-\infty}$ and $u d^{\infty} \neq d^{-\infty}$ (recall that we denote $g^{-\infty}=\lim _{n \rightarrow+\infty} g^{-n}$ for $g$ of infinite order). Consider $x_{k}=c^{k} u c^{k}$ and $y_{k}=d^{k} u d^{k}$. For $k$ large, the above inequalities imply that these two elements have infinite order, and do not generate a virtually cyclic group because $x_{k}{ }^{+\infty}$ and $x_{k}{ }^{-\infty}$ (respectively $y_{k}{ }^{+\infty}$ and $y_{k}{ }^{-\infty}$ ) is close to $c^{+\infty}$ and $c^{-\infty}$ ( respectively $d^{+\infty}$ and $d^{-\infty}$ ). Fix $k$, and consider the elements $z_{n}=x_{k}{ }^{n+1} y_{k}{ }^{-n}$. They belong to the coset $C$, because $\Gamma / K$ is abelian, and their stable norm goes to infinity with $n$. Therefore $C$ contains infinitely many conjugacy classes. Let us now construct $c, d$ as above. Choose $a, b \in K$ generating a free group of rank 2 . 
We first explain how to get $c$. There is a problem only if $u a^{\infty}=a^{-\infty}$ and $u b^{\infty}=b^{-\infty}$. In that case there exists integers $p, q$ with $u a^{p} u^{-1}=a^{-p}$ and $u b^{q} u^{-1}=b^{-q}$. We take $c=a^{p} b^{q}$, noting that $u c u^{-1}=a^{-p} b^{-q}$ is different from $c^{-1}=b^{-q} a^{-p}$.

Once we have $c$, we choose $c^{*} \in K$ with $\left\langle c, c^{*}\right\rangle$ free of rank 2 , and we obtain $d$ by applying the preceding argument using $c^{*}$ and $c c^{*}$ instead of $a$ and $b$. The group $\langle c, d\rangle$ is free of rank 2 because $d$ is a positive word in $c^{*}$ and $c c^{*}$.

Theorem 6.4 If $G_{\varphi}$ has a non-elementary action by isometries on a Gromov-hyperbolic length space, then $G$ has infinitely many $\varphi$-twisted conjugacy classes.

Proof. By elementary action we mean an action consisting of elliptic elements, or with a global fixed point, or with a global fixed pair in the boundary of the hyperbolic space. The statement of the theorem immediately follows from Lemma 6.2 and Delzant Lemma 6.3.

The Theorem 6.4 applies if $G$ is a Gromov-hyperbolic group and $\varphi$ has finite order in $\operatorname{Out}(G)$. In fact in this case, $G_{\varphi}$ contains $G$ as a subgroup of finite index, thus is quasi-isometric to $G$, and by quasi-isometry invariance, it is itself Gromov-hyperbolic group.

The Theorem 6.4 also applies when $G$ is so-called relatively hyperbolic and $\varphi$ is of finite order in $\operatorname{Out}(G)$. In this case, the quasi-isometry invariance of $G_{\varphi}$ and $G$ is harder to establish, but is has been proven by Druţu in [8].

Remark. For automorphism of a hyperbolic group, of infinite order in $\operatorname{Out}(G)$, the fact that $G$ has infinitely many $\varphi$-twisted conjugacy classes was implicitly proven by Levitt and Lustig in [39], see also [13]. Similarly we can prove that $R(\varphi)=\infty$ for automorphism $\varphi$ of infinite order in $\operatorname{Out}(G)$, where $G$ is relatively hyperbolic group.

Let now $S$ be an oriented compact surface with genus $g$ and with $p$ boundary components, where $3 g+p-4>0$. It is easy to see that the mapping class group $\operatorname{Mod}_{S}$ is a normal subgroup of the full mapping class group $\operatorname{Mod}_{S}^{*}$, of index 2. The graph of curves of $S$, denoted $\mathcal{G}(S)$, is the graph whose vertices are the simple curves of $S$ modulo isotopy. Two vertices (that is 
two isotopy classes of simple curves) are linked by an edge in this graph if they can be realized by disjoint curves. Both $\operatorname{Mod}_{S}$ and $\operatorname{Mod}_{S}^{*}$ act on $\mathcal{G}(S)$ in a non-elementary way.

Thus, Theorem 6.4 is applicable for $\operatorname{Mod}_{S}$ and for $\varphi_{1}$ the automorphism induced by reversing the orientation of $S$, since in this case, $\left(\operatorname{Mod}_{S}\right)_{\varphi_{1}}=$ $\operatorname{Mod}_{S} \rtimes_{\varphi_{1}} \mathbb{Z}_{2} \simeq \operatorname{Mod}_{S}^{*}$. For $\operatorname{Mod}_{S}$ and $\varphi_{0}=I d$ we have $R\left(\varphi_{0}=I d\right)=\infty$ because the group $\operatorname{Mod}_{S}$ has infinite number of usual conjugacy classes. Finally, $\operatorname{Out}\left(\operatorname{Mod}_{S}\right) \simeq\left\{\overline{\varphi_{0}}, \overline{\varphi_{1}}\right\}$ see [34], which by Lemma 2.1 ensures that $\operatorname{Mod}_{S}$ has property $R_{\infty}$ if $S$ is an orientable compact surface of genus $g$ with $p$ boundary components, where $3 g+p-4>0$. The only cases not covered by this inequality are where $\mathrm{S}$ is the torus with at most one hole or where $\mathrm{S}$ is the sphere with at most 4 holes. The case of a torus with at most one hole follows from the section 3 . The case of the sphere with at most 4 holes follows directly from the knowledge of Out(ModS) and the cardinality of the mapping class group.

\section{REFERENCES}

1. J. Arthur and L. Clozel, Simple algebras, base change, and the advanced theory of the trace formula, Princeton University Press, Princeton, NJ, 1989. MR90m:22041

2. G. Baumslag and D. Solitar, Some two-generator one-relator non-hopfian groups, Bull. Am. Math. Soc. 68 (1962), 199-201.

3. J. S. Birman, Braids, links and mapping class groups, Ann. Math. Stud. 82, Princeton University Press, 1974.

4. C. Bleak, A. Fel'shtyn, and D. L. Gonçalves, Twisted conjugacy classes in R. Thompson's group F, E-print arXiv:math.GR/0704.3441, 2007

5. B. Bowditch, Intersection numbers and the hyperbolicity of the curve complex, J. Reine Angew.Math.(Crelles Journal) 598(2006), 105-131.

6. K. Brown, Cohomology of Groups, Springer verlag 82, New York, 1982.

7. D.Cohen, Combinatorial group theory: a topological approach, LMS Student Text 14, Cambridge University Press, 1989.

8. C Druţu, Relatively hyperbolic groups: geometry and quasi-isometric invariance. Eprint arXiv: math.GR/0605211.

9. J. L. Dyer and E. K. Grossman, The automorphism groups of the braid groups, Amer. J. of Math 103 no.6 (1981), 1151-1169.

10. B. Farb and L. Mosher, Quasi-isometric rigidity for solvable Baumslag-Solitar groups, II, Invent. Math. 137 no.3 (1999), 613-649.

11. B. Farb and L. Mosher, On the asymptotic geometry of abelian-by-cyclic groups, Acta Math. 184 no.2 (2000), 145-202. 
12. A. Fel'shtyn, Dynamical zeta functions, Nielsen theory and Reidemeister torsion, Mem. Amer. Math. Soc. 147 (2000), no. 699, xii+146. MR2001a:37031

13. A. L. Fel'shtyn, The Reidemeister number of any automorphism of a Gromov hyperbolic group is infinite, Zapiski Nauchnych Seminarov POMI 279 (2001), 229-241.

14. A. L. Fel'shtyn and D. L. Gonçalves, Reidemeister numbers of any automorphism of Baumslag-Solitar group is infinite. Geometry and Dynamics of Groups and Spaces, Progress in Mathematics, v. 265(2008), 286-306, Birkhauser.

15. A. L. Fel'shtyn, D. L. Gonçalves and P. Wong, Twisted conjugacy classes of automorphisms of Polyfree groups, in preparation.

16. A. L. Fel'shtyn and R. Hill, The Reidemeister zeta function with applications to Nielsen theory and a connection with Reidemeister torsion, K-theory 8 no.4 (1994), $367-393$.

17. A. Fel'shtyn and E. Troitsky, Geometry of Reidemeister classes and twisted Burnside theorem, Journal of K-theory 1(2008), 1-40..

18. A. Fel'shtyn and E. Troitsky, Twisted Burnside - Frobenius theory for discrete groups, ( to appear in Crelle Journal).

19. A. L. Fel'shtyn and E. Troitsky, Twisted Burnside theorem for countable groups and Reidemeister numbers, Noncommutative Geometry and Number Theory, Vieweg, Braunschweig, 2006, 141-154.

20. A. Fel'shtyn, E. Troitsky, and A. Vershik, Twisted Burnside theorem for type $\mathrm{II}_{1}$ groups: an example, Mathematical Research Letters 13(2006), no.5, 719-728.

21. A. Fel'shtyn,Y. Leonov, E. Troitsky, Reidemeister numbers of saturated weakly branch groups. E-print arXiv: math.GR/0606725 Preprint MPIM 2006-79. (to appear in Geometria Dedicata).

22. N. D. Gilbert, J. Howie, V. Metaftsis and E. Raptis, Tree actions of automorphism group, J. Group Theory 2 (2000), 213-223.

23. D. L. Gonçalves, The coincidence Reidemeister classes on nilmanifolds and nilpotent fibrations, Top. and its Appl. 83 (1998), 169-186.

24. D. L. Gonçalves and J. Guaschi, The lower central and derived series of the braid groups $B_{n}\left(S^{2}\right)$ and $B_{m}\left(S^{2} \backslash\left\{x_{1}, \ldots, x_{n}\right\}\right)$, preprint, March 2006.

25. D. L. Gonçalves and P. Wong, Twisted conjugacy classes in exponential growth groups, Bulletin of the London Mathematical Society 35 (2003), 261-268.

26. D. L. Gonçalves and P. Wong, Homogeneous spaces in coincidence theory II, Forum 17 (2005), 297-313.

27. D. L. Gonçalves and P. Wong, Twisted conjugacy classes in wreath products, Internat. J. Alg. Comput. 16 No. 5 (2006), 875-886.

28. M. Gromov, Groups of polynomial growth and expanding maps. Publicationes Mathematiques, 53, 1981, 53-78.

29. M. Gromov, Hyperbolic groups, in: S. Gersten, ed., "Essays in Group Theory" MSRI Publications 8 Springer-Verlag, Berlin, New York, Heidelberg, 1987, 75-263. 
30. A. Grothendieck, Formules de Nielsen-Wecken et de Lefschetz en géométrie algébrique, Séminaire de Géométrie Algébrique du Bois-Marie 1965-66. SGA 5, Lecture Notes in Math., vol. 569, Springer-Verlag, Berlin, 1977, pp. 407-441.

31. P. de la Harpe, "Topics in Geometric Group Theory," Chicago Lectures in Mathematics Series, The Unversity of Chicago Press, Chicago, 2000.

32. L. K. Hua and I. Reiner, Automorphisms of the unimodular group, Trans. Amer. Math. Soc. 71 (1951), 331-348.

33. L. K. Hua and I. Reiner, Automorphisms of the projective unimodular group, Trans. Amer. Math. Soc. 72 (1952), 467-473.

34. N. V. Ivanov, Automorphisms of Teichm uller modular groups, Lecture Notes in Mathematics 1346 Springer, Berlin, 1988.

35. B. Jiang, Lectures on Nielsen fixed point theory, Contemp. Math., vol. 14, Amer. Math. Soc., Providence, RI, 1983.

36. M. Kapovich, "Hyperbolic Manifolds and Discrete Groups," Birkhäuser, Boston, Basel, Berlin, 2000.

37. D. Kochloukova, Injective endomorphisms of the Baumslag-Solitar group, Algebra Colloquium 13 no3 (2006), 525-534.

38. G. Levitt, On the automorphism group of generalised Baumslag-Solitar groups, Eprint arxiv:math.GR/0511083, 2005.

39. G. Levitt and M. Lustig, Most automorphisms of a hyperbolic group have very simple dynamics, Ann. Scient. Éc. Norm. Sup. 33 (2000), 507-517.

40. W. Magnus, A. Karrass and D. Solitar, Combinatorial Group Theory. New York: Interscience Publishers, (1966).

41. H. A. Masur, Y. N. Minsky, Geometry of the complex of curves I: hyperbolicity, Invent. Math. 138 (1999), 103-149.

42. M. Newmann, Integral matrices, Academic Press, New York and London, 1972.

43. K. Reidemeister, Automorphismen von Homotopiekettenringen, Math. Ann. 112 (1936), 586-593.

44. Irving Reiner, Automorphisms of the symplectic modular group, Trans. Amer. Math. Soc. 80 (1955), 35-50.

45. G. P. Scott, Braid groups and the group of homeomorphisms of a surface, Proc. Camb. Phil. Soc., 68 (1970), 605-617.

46. Salahoddin Shokranian, The Selberg-Arthur trace formula, Lecture Notes in Mathematics, vol. 1503, Springer-Verlag, Berlin, 1992, Based on lectures by James Arthur. MR1176101 (93j:11029)

47. Jennifer Taback and Peter Wong, Twisted conjugacy and quasi-isometry invariance for generalized solvable Baumslag-Solitar groups, E-print arxiv:math.GR/0601271, 2006.

48. Jennifer Taback and Peter Wong, A note on twisted conjugacy and generalized Baumslag-Solitar groups, E-print arXiv:math.GR/0606284, 2006.

49. E. Troitsky, Noncommutative Riesz theorem and weak Burnside type theorem on twisted conjugacy, Funct. Anal. Pril. 40 (2006), no. 2, 44-54, In Russian, English 
translation: Funct. Anal. Appl. 40 (2006), No. 2, 117-125 (Preprint 86 (2004), MaxPlanck-Institut für Mathematik, math.OA/0606191).

50. P. Wong, Fixed-point theory for homogeneous spaces, Amer. J. Math. 120 (1998), $23-42$.

Instytut Matematyki, Uniwersytet Szczecinski, Ul. Wielkopolska 15, 70451 Szczecin, Poland and Boise State University, 1910 University Drive, Boise, IDAHO, 83725-155, USA

E-mail address: felshtyn@diamond.boisestate.edu, felshtyn@mpim-bonn.mpg.de

Dept. de Matemática - IME - USP, Caixa Postal 66.281 - CeP 05311-970, SÃo PAUlo - SP, BRASIL

E-mail address: dlgoncal@ime.usp.br

Francois Dahmani, Laboratoire Emile Picard, Umr CNRS 5580, Université Paul Sabatier, 31062 Toulouse Cedex 4, France

E-mail address: francois.dahmani@math.ups-tlse.fr 